\title{
Dynamic analysis of a parametrically excited golden Muga silk embedded pneumatic artificial muscle
}

\author{
Bhaben Kalita ${ }^{1, *}$ and S. K. Dwivedy,"* \\ ${ }^{1}$ Mechanical Engineering Department, Indian Institute of Technology Guwahati, Guwahati, 781039, \\ India
}

\begin{abstract}
In this work a novel pneumatic artificial muscle is fabricated using golden muga silk and silicon rubber. It is assumed that the muscle force is a quadratic function of pressure. Here a single degree of freedom system is considered where a mass is supported by a spring-damper-and pneumatically actuated muscle. While the spring-mass damper is a passive system, the addition of pneumatic muscle makes the system active. The dynamic analysis of this system is carried out by developing the equation of motion which contains multi-frequency excitations with both forced and parametric excitations. Using method of multiple scales the reduced equations are developed for simple and principal parametric resonance conditions. The time response obtained using method of multiple scales have been compared with those obtained by solving the original equation of motion numerically. Using both time response and phase portraits, variation of few systems parameters have been carried out. This work may find application in developing wearable device and robotic device for rehabilitation purpose.
\end{abstract}

\section{Introduction}

A wearable device used in rehabilitation robotic system guides the human motion in a similar way as that of the function of the human muscles. For this purpose, the used actuators should have a high power-to-weight ratio so that the device or the robot must be of light weight. To achieve this goal, it is necessary to develop a pneumatic artificial muscle (PAM) actuator that is soft in nature similar to human muscles. The main advantages of the artificial muscle are; its high power-to-weight ratio, low price, low maintenance and capabilities for use in rough environments. Basically, PAMs are contractile and linear motion actuators operated by gas pressure. The force and motion generated by this type of actuators are linear and unidirectional.

The McKibben-type artificial muscles [1-4] are now widely used. However, the physical model of this type of PAMs is highly nonlinear [5-7] and the generated heat and mechanical loss are considerable because of the friction caused by expansion and contraction of the sleeve. For this purpose, the product life of this actuator is shortened greatly by friction. A modified PAM based on the McKibben type has been produced by Festo [8] which is also highly nonlinear in nature, but it has a high tensile strength and a very long lifetime.

As an alternative, a novel PAM is proposed in this work, in which silicon rubber is embedded with the high-intensity locally available fabrics like Golden Muga silk. These

\footnotetext{
*Corresponding author: k.bhaben@iitg.ernet.in, dwivedy@iitg.ernet.in
} 
PAMs are one such class of actuators in which fabrics are arranged longitudinally to make it frictionless so that the product life cycle can be extended and hysteresis of the actuator becomes small. These will be capable of delivering high power-to-weight ratio just like the McKibben Muscle and significant similarities with our body muscles. However the effect of their variably controlled stiffness on system dynamics has yet to be understood and forms the purpose of this paper.

The artificial muscle is model as a single degree of freedom system where the actuator force is considered as a function of pressure for a particular length, thickness, and material properties of the used muscle. Following experimental observations of similar type of PAM, a quadratic relation between the muscular tension and the internal pressure is assumed in this work. Also, the variation of pressure in the muscle is assumed to be a periodically time varying pressure. Hence the derived resulting governing equation of motion is in the form of a forced and parametrically excited system. The approximate analytical solution for the developed equation is then formulated by applying the first order method of multiple scales. These solutions can be easily used for finding the system response instead of directly numerically solving the governing differential equation of motion. The parametric instability regions have been determined which will help the designer to specify the safe operating range of system parameters.

\section{Fabrication of the proposed pneumatic artificial muscle (PAM)}

Figure 1 shows the artificial muscle developed using silicone rubber and golden muga silk. Initially, threads of muga silk have been made to align in the axial direction by using an apparatus develop for this purpose. A plastic mould has been prepared to fabricate the PAM which is of the cylindrical shape. The liquid silicone rubber was poured into the mould containing the muga silk threads and kept for around 24 hours in natural environment for curing. The developed PAM is shown in Fig. 1 with the necessary attachment for supplying air pressure. It may be noted that the number of threads, thickness of the rubber, length and radius of the PAM can be changed to achieve the required property for a particular application. By applying pneumatic pressure as the length is constant due to muga fabrics, the longitudinal and lateral expansion can be controlled.

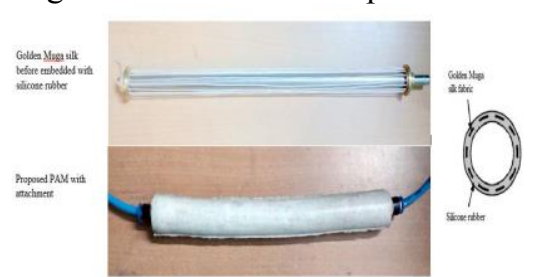

Figure 1. Proposed artificial pneumatic muscle.

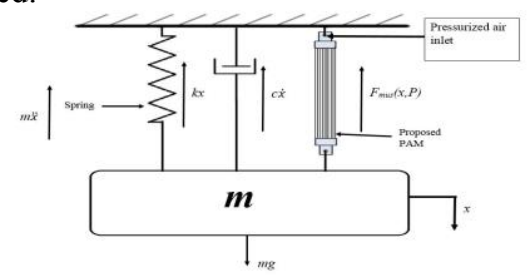

Figure 2. Schematic diagram of a system actuated using Proposed PAM.

\section{System modelling}

Figure 2 shows a system containing a spring with stiffness $k$, a damper with coefficient of viscous damping $c$ and a pneumatically actuated muscle connected to a mass $m$. Such a system may be useful for industrial and rehabilitation robotics purpose.

The equation of motion of the system may be derived using Newton's $2^{\text {nd }}$ law and can be written as

$$
m \ddot{x}+k x+c \dot{x}+F_{m u s}-m g=0
$$


where the dots denote differentiation with respect to time $t$ and $x$ is the displacement with respect to the unstressed position of the spring and PAM. $F_{m u s}$ is the force exerted by the PAM, and $g$ denotes the acceleration due to gravity. The expression for the muscle force $F_{m u s}$ is assumed to be similar to that given in the work of Hongbing et al. [9].

$$
F_{\text {mus }}(x, P)=\left(c_{1}+c_{2} P+c_{3} P^{2}\right)\left(\frac{x}{l_{\max }}\right)+\left(d_{1}+d_{2} P\right)
$$

where $c_{1}, c_{2}, c_{3}, d_{1}$ and $d_{2}$ are constants which should be determined from experiments and $l_{\max }$ is the maximum possible muscle length. Thus the system equation of motion can be expanded by combining Eq. (1) and (2) to yield

$$
\ddot{x}+\left\lfloor\frac{k}{m}+\frac{\left(c_{1}+c_{2} P+c_{3} P^{2}\right)}{m l_{\max }}\right\rfloor x+\frac{c}{m} \dot{x}+\left[\frac{\left(d_{1}+d_{2} P\right)}{m}-g\right]=0
$$

To study the dynamics of the system in a realistic condition, a harmonics internal pressure input is assumed for the muscle in the form of

$$
P=P_{m}+P_{0} \sin \bar{\Omega} t
$$

This approximation is similar to the work of [9-11]. In practice, this may be implemented using a PI controller and proportional flow control valves [12].

Now considering a non-dimensional time $\tau=\omega_{0} t$, where $\omega_{0}$ is the fundamental natural frequency of the system which is given by

$$
\omega_{0}=\sqrt{(k / m)+\left(c_{1}+c_{2} P_{m}+c_{3}\left(P_{m}^{2}+0.5 P_{0}^{2}\right)\right) /\left(m l_{\max }\right)}
$$

Using the following non-dimensional parameters

$$
\Omega=\frac{\bar{\Omega}}{\omega_{0}}, \mu=\frac{c}{2 \varepsilon m \omega_{0}}, p_{1}=\frac{c_{2} P_{0}+2 c_{3} P_{0} P_{m}}{\varepsilon m \omega_{0}^{2} l_{\max }}, p_{2}=-\frac{c_{3} P_{0}^{2}}{2 \varepsilon m \omega_{0}^{2} l_{\max }}, f_{1}=\frac{d_{2} P_{m}+d_{1}-m g}{\varepsilon m \omega_{0}^{2}}, f_{2}=\frac{d_{2} P_{0}}{\varepsilon m \omega_{0}^{2}}
$$

Eq. (3) can be simplified to the form

$$
\ddot{x}+x+2 \varepsilon \mu \dot{x}+\in\left(p_{1} \sin \Omega \tau+p_{2} \cos 2 \Omega \tau\right) x=\varepsilon\left(f_{1}+f_{2} \sin \Omega \tau\right)
$$

where the dots indicate the differentiation with respect to $\tau$ and $\varepsilon$ is a dimensionless book keeping parameter which is less than 1 and $\mu$ is the non-dimensional damping factor. It may be noted that the nondimentional parameters $p_{1}, p_{2}$ and $f_{2}$ are function of static pressure $P_{0}$. Also, $p_{1}$ and $f_{1}$ are the function of dynamic pressure $P_{m}$. From the fourth term on the left hand side of Eq. (7), it may clearly be observed that the coefficients of response $x$ contains time varying terms with frequency $\Omega$ and $2 \Omega$. Hence this is a parametrically excited system with multi frequency excitation. In addition to this the system is also a forced vibration system having a sinusoidally varying force of amplitude $f_{2}$ and frequency $\Omega$. The solution to this equation is studied using first order method of multiple scales in the following sections.

The approximate solution of the above equation is obtained by using the first order method of multiple scales.

$$
\begin{gathered}
x(\tau ; \varepsilon)=x_{0}\left(T_{0}, T_{1}\right)+\varepsilon x_{1}\left(T_{0}, T_{1}\right) \\
\text { where, } T_{n}=\varepsilon^{n} \tau, T_{0}=\tau, T_{1}=\varepsilon \tau \text { and } D_{0}=\frac{d}{d \tau}, D_{1}=\varepsilon \frac{d}{d \tau}
\end{gathered}
$$

Here the simple resonance condition of the system is considered when the nondimentional external excitation frequency $\Omega \cong 1$. Introducing the detuning 
parameter $\sigma$ one may write $\Omega=1+\in \sigma$. Following the standard procedure of method of multiple scales, the reduced equations obtained in this case are given below.

$$
\begin{aligned}
& \frac{d a}{d t}=\varepsilon\left\{\left(-\frac{f_{2}}{2}\right) \cos \gamma-\mu a-\frac{p_{2}}{4} a \sin 2 \gamma\right\} \\
& \frac{d \gamma}{d t}=\varepsilon \sigma-\left\{\varepsilon\left(-\frac{f_{2}}{2 a}\right) \sin \gamma+\frac{p_{2}}{4} \cos 2 \gamma\right\}
\end{aligned}
$$

The final response of the system can be given by solving Eq. (9), Eq. (10); and using it in the following equation.

$$
\begin{aligned}
& x(\tau)=a\left(T_{1}\right) \cos \left(\Omega T_{0}-\gamma\left(T_{1}\right)\right)+\varepsilon\left(f_{1}-\frac{p_{1} a\left(T_{1}\right)}{2\left(1-(1-\Omega)^{2}\right)} \sin \gamma\left(T_{1}\right)\right) \\
& +\varepsilon\left(-\frac{p_{1} a\left(T_{1}\right)}{2\left(1-(1+\Omega)^{2}\right)} \sin \left(2 \Omega T_{0}-\gamma\left(T_{1}\right)\right)-\frac{p_{2} a\left(T_{1}\right)}{2\left(1-(1+2 \Omega)^{2}\right)} \cos \left(3 \Omega T_{0}-\gamma\left(T_{1}\right)\right)\right)
\end{aligned}
$$

Eq. (11) gives the expression for the time response of the system for the simple resonance condition.

Next considering the principal parametric resonance condition of the system where, one may introduce the detuning parameter $\sigma$ as $\Omega=2+\varepsilon \sigma$ and following similar procedure, the reduced equations can be given by

$$
\begin{gathered}
\frac{d a}{d t}=\varepsilon\left(-\mu a+\frac{p_{1}}{4} a \cos 2 \gamma\right) \\
\frac{d \gamma}{d t}=\frac{\varepsilon \sigma}{2}-\varepsilon\left(\frac{p_{1}}{4} \sin 2 \gamma\right)
\end{gathered}
$$

Clearly it shows that the system has a trivial state response which may be unstable for some system parameters and the response can be given by the following equation,

$$
\begin{aligned}
& x(\tau)=a\left(T_{1}\right) \cos \left(\frac{\Omega T_{0}-2 \gamma\left(T_{1}\right)}{2}\right)+\varepsilon\left(f_{1}+\frac{f_{2}}{(1-\Omega)^{2}} \sin \Omega T_{0}-\frac{p_{1} a\left(T_{1}\right)}{2\left(1-(1+\Omega)^{2}\right)} \sin \left(\frac{3 \Omega T_{0}-2 \gamma\left(T_{1}\right)}{2}\right)\right) \\
& +\varepsilon\left(-\frac{p_{2} a\left(T_{1}\right)}{2\left(1-(1+2 \Omega)^{2}\right)} \cos \left(\frac{5 \Omega T_{0}-2 \gamma\left(T_{1}\right)}{2}\right)-\frac{p_{2} a\left(T_{1}\right)}{2\left(1-(2 \Omega-1)^{2}\right)} \cos \left(\frac{3 \Omega T_{0}+2 \gamma\left(T_{1}\right)}{2}\right)\right)
\end{aligned}
$$

Eq. (14) gives the complete time response of the system for the principal parametric resonance condition.

\section{Numerical results and discussion}

Considering the system parameters of the PAM as given in Table 1 the numerical simulations has been carried out to determine the time response and phase portrait of the system shown in Fig. 2 for simple and principal parametric resonance condition.

Table 1. Numerical values for system parameters.

\begin{tabular}{|c|c|c|c|c|c|}
\hline Parameter & $\begin{array}{c}\text { Numerical } \\
\text { Value }\end{array}$ & Parameter & $\begin{array}{c}\text { Numerical } \\
\text { Value }\end{array}$ & Parameter & $\begin{array}{c}\text { Numerical } \\
\text { Value }\end{array}$ \\
\hline$l_{\max }$ & $74 \mathrm{~mm}$ & $d_{1}$ & $-100 \mathrm{~N}$ & $P_{0}$ & $7 \mathrm{kPa}$ \\
\hline$c_{1}$ & $-234.25 \mathrm{~N}$ & $d_{2}$ & $1 \mathrm{~N} / \mathrm{kPa}$ & $P_{m}$ & $30 \mathrm{kPa}$ \\
\hline$c_{2}$ & $1.96 \mathrm{~N} / \mathrm{kPa}$ & $m$ & $6 \mathrm{~N}$ & $\mu$ & 0.02 \\
\hline$c_{3}$ & $-0.003 \mathrm{~N} / \mathrm{kPa}^{2}$ & $k$ & $12 \mathrm{~N} / \mathrm{mm}$ & $\varepsilon$ & 0.1 \\
\hline
\end{tabular}




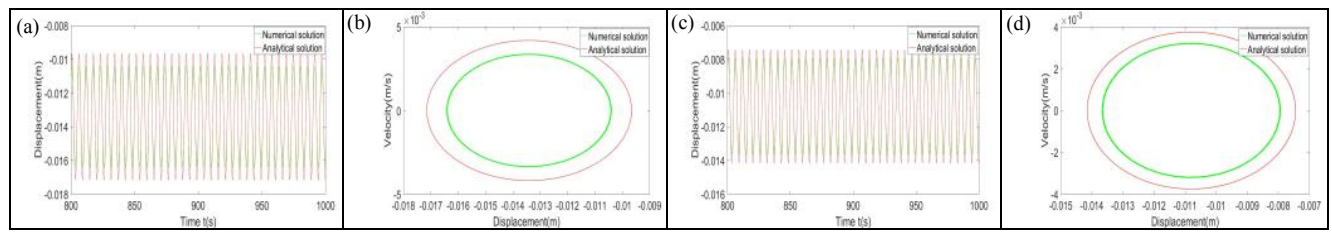

Figure 3. Time response and phase portrait for different values of $k, P_{0}, P_{m}$ at simple resonance condition $(\mathrm{a}, \mathrm{b}) k=12 \mathrm{kN} / \mathrm{m}, P_{0}=7 \mathrm{kPa}, P_{m}=30 \mathrm{kPa} ;(\mathrm{c}, \mathrm{d}) k=12 \mathrm{kN} / \mathrm{m}, P_{0}=7 \mathrm{kPa}, P_{m}=50 \mathrm{kPa}$

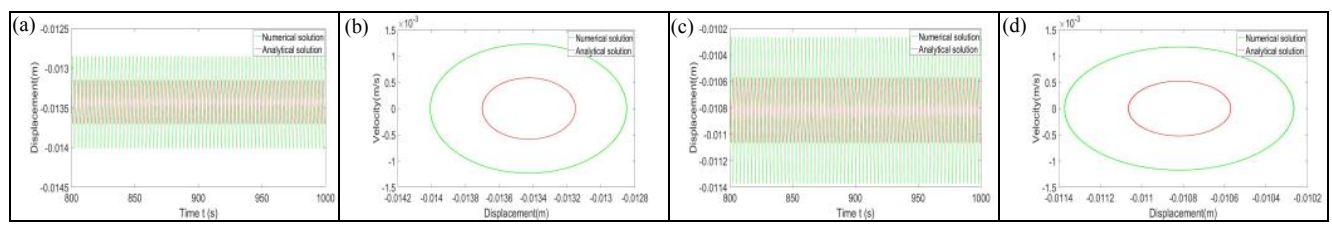

Figure 4. Time response and phase portrait for different values of $k, P_{0}, P_{m}$ for principal parametric resonance condition (a, b) $k=12 \mathrm{kN} / \mathrm{m}, P_{0}=7 \mathrm{kPa}, P_{m}=30 \mathrm{kPa}$; (c, d) $k=12 \mathrm{kN} / \mathrm{m}, P_{0}=7 \mathrm{kPa}, P_{m}=50 \mathrm{kPa}$

For simple resonance condition the time responses and phase portraits are shown in Figure 3 by changing $P_{m}$ from $30 \mathrm{kPa}$ to $50 \mathrm{kPa}$. Similarly for principal parametric resonance conditions these responses are shown in Fig.4. By varying different system parameters and finding the time responses, 5 cases have been studied as given in Table 2 . In the first case taking $k=12 \mathrm{~N} / \mathrm{mm}, P_{0}=7 \mathrm{kPa}, P_{m}=30 \mathrm{kPa}, \mu=0.02$ the time response and phase portrait are shown in Fig. 3(a,b). It may clearly be observed that the solution obtain by solving the original equation Eq. (7) and those obtain by method of multiple scale Eq. (11) are in good agreement. In this case the response amplitude is obtained to be $13.37 \mathrm{~mm}$ from analytical solution and $13.38 \mathrm{~mm}$ from numerical solution. Now by changing only $P_{m}$ from $30 \mathrm{kPa}$ to $50 \mathrm{kPa}$ the mean displacement in Fig. 3(c, d) is decreased to $10.8 \mathrm{~mm}$ which $19.22 \%$ from $1^{\text {st }}$ case. Similarly by changing only $P_{0}$ from $7 \mathrm{kPa}$ to $70 \mathrm{kPa}$ the mean displacement is decrease to $11.13 \mathrm{~mm}$ which $16.83 \%$ from $1^{\text {st }}$ case. Again by changing stiffness $k$ from $12 \mathrm{~N} / \mathrm{mm}$ to $8 \mathrm{~N} / \mathrm{mm}$ the mean displacement is increased to $22.86 \mathrm{~mm}$ which $70.98 \%$ from the case 1 . Finally keeping all the system parameters same except the damping parameter $\mu$ which is changed from 0.02 to 0.1 , the mean response amplitude is $13.40 \mathrm{~mm}$ which is almost equal to that of case 1 .

For principal parametric resonance condition the results have been obtained for the same 5 different system parameters as given in Fig. 4 and Table 2. In the first case taking $k=12 \mathrm{~N} / \mathrm{mm}, P_{0}=7 \mathrm{kPa}, P_{m}=30 \mathrm{kPa}, \mu=0.02$ the time response and phase portrait are shown in Fig. $4(a, b)$. It may clearly be observed that the solution obtain by solving the original equation Eq. (7) and those obtain by method of multiple scale Eq. (14) are in good agreement. In this case the response amplitude is obtained to be $13.43 \mathrm{~mm}$ from the analytical solution and for numerical solution also it is same. Now by changing only $P_{m}$ from $30 \mathrm{kPa}$ to $50 \mathrm{kPa}$ the mean displacement in Fig. 4 (c,d) is decreased to $10.82 \mathrm{~mm}$ which is $19.44 \%$. Similarly by changing only $P_{0}$ from $7 \mathrm{kPa}$ to $70 \mathrm{kPa}$ the mean displacement is slightly decrease to $13.30 \mathrm{~mm}$ which $0.96 \%$ from the case 1 . Now by changing stiffness $k$ from $12 \mathrm{~N} / \mathrm{mm}$ to $8 \mathrm{~N} / \mathrm{mm}$ the mean displacement is increased to $23.02 \mathrm{~mm}$ which is $71.41 \%$ from case 1 . Finally keeping all the system parameters same except the damping parameter $\mu$ which is changed from 0.02 to 0.1 the mean response amplitude is $13.43 \mathrm{~mm}$ 
which is equal to case 1 . Hence for different applications one may obtained the required displacement by changing different system parameters of the muscles which can be obtained easily by using the developed reduced equations.

Table 2. Comparison of the response amplitude for different resonance conditions.

\begin{tabular}{|c|c|c|c|c|c|c|c|c|}
\hline \multirow{3}{*}{ Observation } & \multicolumn{4}{|c|}{ System parameter } & \multirow{2}{*}{\multicolumn{2}{|c|}{$\begin{array}{c}\text { Simple } \\
\text { resonance } \\
\text { condition }\end{array}$}} & \multirow{2}{*}{\multicolumn{2}{|c|}{$\begin{array}{l}\text { Prin. parametric } \\
\text { resonance } \\
\text { condition }\end{array}$}} \\
\hline & \multirow{2}{*}{$\begin{array}{c}k \\
(\mathrm{~N} / \mathrm{mm})\end{array}$} & \multirow{2}{*}{$\begin{array}{c}P_{0} \\
(\mathrm{kPa})\end{array}$} & \multirow{2}{*}{$\begin{array}{c}P_{m} \\
(\mathrm{kPa})\end{array}$} & \multirow{2}{*}{$\mu$} & & & & \\
\hline & & & & & $\begin{array}{l}\text { Mean } \\
(\mathrm{mm})\end{array}$ & $\begin{array}{l}\text { Amp. } \\
\text { (mm) }\end{array}$ & $\begin{array}{l}\text { Mean } \\
(\mathrm{mm})\end{array}$ & $\begin{array}{l}\text { Amp. } \\
\text { (mm) }\end{array}$ \\
\hline Case1 & 12 & 7 & 30 & 0.02 & 13.37 & 3.70 & 13.43 & 0.58 \\
\hline Case2 & 12 & 7 & 50 & 0.02 & 10.80 & 3.35 & 10.82 & 0.56 \\
\hline Case 3 & 12 & 70 & 30 & 0.02 & 11.13 & 35.22 & 13.30 & 6.14 \\
\hline Case4 & 8 & 7 & 30 & 0.02 & 22.86 & 7.45 & 23.02 & 1.01 \\
\hline Case5 & 12 & 7 & 30 & 0.1 & 13.40 & 2.83 & 13.43 & 0.58 \\
\hline
\end{tabular}

\section{Conclusion}

In this work a single degree of freedom of system containing spring-mass damper system along with a pneumatic artificial muscle is considered. The dynamic analysis of this active system is carried out using method of multiple scales. Two resonance conditions viz., simple resonance condition and principal parametric resonance conditions have been carried out and the time resonance and phase portraits have been found. The mean value of the numerical and analytical results is found to be in good agreement. Hence, this formulation may be used for calculating the response of the system for different pressures which will form the basis for developing the controller for different applications. It may be noted that by changing the dimensions of the thread, number of thread, length of thread for different dimension of the artificial muscles, after obtaining the parameters of the muscle force- pressure equation, one may easily find the response of the system which will be suitable for many different applications.

\section{References}

[1] V. L. Nickel, J. Perry, A. L. Garrett, J. Bone Joint Surg., 45A, 933-952 (1963).

[2] M. M. Gavrilovic, M. R. Maric, Med. Biol. Eng., 7, 77-82 (1969).

[3] G. K. Klute, J. M. Czernieki, B. Hannaford, in: Proc. IEEE/ASME Int. Conf. on Advanced Intelligent Mechatronics, Atlanta, GA, pp. 221-226 (1999).

[4] C. P. Chou, B. Hannaford, in: Proc. IEEE Int. Conf. on Robotics and Automation, San Diego, CA, pp. 281-286(1994).

[5] D. G. Caldwell, A. Razak, M. Goodwin, in: Proc. IFAC Workshop on Intelligent Autonomous Vehicles, Southampton, pp. 507-512 (1993).

[6] D.W. Repperger, K. R. Johnson, C. A. Philips, in: Proc. American Control Conf., San Diego, CA, pp. 1525-1529 (1999).

[7] C. P. Chou, B. Hannaford, IEEE Trans. Robotics Automat., 12, 90-102 (1996).

[8] Festo AG \& Co., (Fluidic Muscle MAS. Retrieved July 5, 2001).

[9] L. Hongbing, S. Ganguly, S. Nakano, K. Tadano, K. Kawashima, International Conference on Applied Bionics and Biomechanics, 69 (2010).

[10] A.S. Das, J.K. Dutt, K. Ray, Appl. Math. Model., 34, 9, 2353-2369 (2010).

[11] M. Azadi, S. Behzadipour, G. Faulkner, JSV, 330, 12, 2733-2746 (2011).

[12] S. Wakimoto, K. Suzumori, T. Kanda, IEEE/RSJ International Conference on Intelligent Robots and Systems, 487-492 (2005). 